Von Jens Clausen ie lokale Agenda soll die Beiträge erarbeiten und bïndeln, die auf kommunaler Ebene für eine nachhaltige Entwicklung geleistet werden können. In einem breiten Konsultationsprozeß sollen daher alle relevanten Akteure einbezogen werden. Von besonderer Bedeutung für die zukünftige Entwicklung von Stadt und Region ist dabei die Wirtschaft mit ihren Unternehmen des Handwerks, des Handels, der mittelständischen und großen Industrie und der Dienstleistung. Die Wirtschaft wäre einerseits von der Umsetzung der Agenda betroffen (z.B. durch Änderung der kommunalen Beschaffungspolitik oder Wirtschaftsförderung) und wird andererseits als aktive Akteurin angesprochen (z.B. Führung von Unternehmen, gerechte Verteilung der Arbeit).

Die Wirtschaft aber, so wird allfallig geklagt, ist nicht wirklich in die Prozesse zur Aufstellung lokaler Agenden 21 eingebunden. Diese Klage kommt von zwei Seiten: von der Wirtschaft selbst, die damit deutlich machen möchte, daß jedwede in diesem Rahmen erzielten Ergebnisse als gesellschaftliche Entscheidungsgrundlage selbstverständlich nicht direkt gültig sind, und von den Initiatoren der Lokalen Agenda 21, die genau dies ebenfalls fürchten. Vor kurzem war deshalb die Frage der Beteiligung der Wirtschaft zentrales Thema eines bundesweiten Kongresses zu den Agenda-Aktivitäten. Sind die Klagen berechtigt?

Ein Blick ins Detail ist, wie so oft, erhellend. So wurden durchaus Firmen und Wirtschaftsverbände zur Partizipation bei der Erstellung der lokalen Agenda 21 als eine von vielen Gruppen eingeladen. Nur aus wenigen Städten ist aber bekannt, daß diese Einladung auch in breiter Form angenommen wurde. Allgemein war wohl die Vorstellung, auf einer Ebene mit Mann oder Frau ,von der Straße" an Zukunftswerkstätten und Planungen teilzunehmen, fuir viele Wirtschaftsvertreter eine kulturelle Überforderung.

Dies hinderte beispielsweise die Wirtschaft in Hannover aber nicht daran, sich Monate nach Abschluß der ersten Arbeitsphase im Rahmen einer Anhörung der Wirtschaft zur Lokalen Agenda 21 bitterlich zu beschweren, daß man keine Gelegenheit zur Mitarbeit bekommen hätte.

Aufschlußreich sind hier die Aussagen einzelner Vertreterlnnen der Wirtschaft zum Endbericht, den die Bürgerbeteiligungsgruppen in Hannover in eineinhalbjähriger Arbeit erarbeitet hatten:
Die Rolle der Wirtschaft in der lokalen Agenda

\section{Mit den Richtigen kooperieren}

Im Rahmen des Agenda 21-Prozesses sind viele Städte und Regionen zur Zeit dabei, sogenannte lokale Agenden aufzustellen. Dabei sollen möglichst alle Akteure vor Ort einbezogen werden. Oft wird bemängelt, daß es an der Beteiligung der Wirtschaft hapert. Doch in seiner Allgemeinheit führt dieser Vorwurf in die Irre. Notwendig ist vielmehr, zwischen verschiedenen Wirtschafitsakteuren zu differenzieren und

Kooperationschancen gezielt zu nutzen.

Ein überregionaler Restaurantbetreiber ließ durchblicken, daß eine lokale Agenda nur dann für die Wirtschaft akzeptabel sei, wenn sie überall gleich sei, denn nur so könne man europaweit disponieren;

eine Autofirma verfiel der in der Wirtschaft üblichen Kurffristigkeit und mahnte an, daß in der langfristigen Agenda Handlungsziele enthalten sind, deren Finanzierung und Umsetzung noch nicht sichergestelt ist, und

ein weltweit agierender Chemiekonzern verwies sehr plausibel auf viele verschiedene Standorte, auf deren Agendapläne man in ihrer Verschiedenheit nur mit lokalen Einzelaktivitäten, aber nicht mit der weltweit gïltigen Unternehmenspolitik reagieren könnte.

\section{Große versus Kleine}

Auch die Industrie- und Handelskammer schloß sich weitgehend den Standpunkten der Meinungsfithrer der Wirtschaft, nämlich den iiberregionalen Konzernen, an und zeigte sich einmal mehr als Vertreterin der Großunternehmen.

Ganz anders die kleinen Unternehmen und die Handwerksunternehmen:

Zwar hatte die Handwerkskammer, die kontinuierlich in den Bürgerbeteiligungsgruppen mitgewirkt hatte, viele Bedenken im Detail, ihre sehr differenzierte Stellungnahme ließ aber auch Synergien zwischen den Schwerpunkten der lokalen Agenda und den Interessen des Handwerks erkennen; eine kleine Tischlerei sah in den ökologischen wie auch den regionalwirtschaftlich orientierten ökonomischen Punkten ausschließlich Riuckenwind für das eigene Unternehmen und

auch ein mittelgroßes Chemieunternehmen fand viel Lob für die Arbeiten der Bürgerbeteiligungsgruppen, sah wohl auch für sich verbesserte Chancen im Falle der Umsetzung der Pläne der Agenda.

Ganz deutlich tut sich hier eine Kluft auf, zwischen den global orientierten Großen und den regional orientierten Kleinen. Ganz deutlich positionierten sich die Großen als Opponenten der lokalen Bürgerbeteiligung überhaupt wie auch der eigenständigen kommunalen Planung und Umsetzung von Maßnahmen im Rahmen der Agenda 21. Aber die von den Großkonzernen hochgehaltene Werte ,Globalisierung" und „weltweiter Wettbewerb" sind für einige kleine und mittlere Unternehmen von deutlich geringerer Bedeutung. Hier ist viel Offenheit und wohl auch die Hoffnung vorhanden, daß lokale Prozesse auch hilfreich für diese Teile der Wirtschaft sein können.

Die lokalen Akteure, die die Agenda 21 vorantreiben, sind also gut beraten, in der Wirtschaft genauso fein wie überall sonst zwischen Unterstützern und Gegnern zu unterscheiden. Dabei sollte besonders kritisch auf die Kammern geachtet werden und darauf, wer hier eigentlich das Wort fihrt. Denn viele kleinere Unternehmen engagieren sich hier kaum, haben auch wohl kaum Zeit dazu.

In jedem Fall scheint klar zu sein: es gibt zumindest Teile der Wirtschaft, die im Rahmen der Agenda 21 kooperieren kömnen und wollen und deren Wachstum vermutlich die Gesellschaft nachhaltiger machen würde. Diese Unternehmen gilt es $\mathrm{zu}$ identifizieren, mit ihnen zu sprechen, zu kooperieren und sie vielleicht zu unterstützen. Und diesen Unternehmen ist zu raten, die Lokale Agenda 21 als Chance zu erkennen, in deren Rahmen insbesondere regionale Kooperationen vorbereitet werden können.

\section{Der Autor}

Jens Clausen ist wissenschafflicher Mitarbeiter im Forschungsfeld Ökologische Unternehmenspolitik am lnstitut für ökologische Wirischaftsforschung. Kontak: lÖW-Projektbüro Hannover, Hausmannstr. $9-10,30159$ Hannover, Tel. 0511/16403-44, Fox -91, E-mail: Jens.Clausen@hannoverioewde 
(c) 20I0 Authors; licensee IÖW and oekom verlag. This is an article distributed under the terms of the Creative Commons Attribution Non-Commercial No Derivates License (http://creativecommons.org/licenses/by-nc-nd/3.o/), which permits unrestricted use, distribution, and reproduction in any medium, provided the original work is properly cited. 